\title{
Self-assembly of iron oxide nanoparticles studied by time-resolved grazing-incidence small-angle x-ray scattering
}

\author{
P. Siffalovic, ${ }^{1}$ E. Majkova, ${ }^{1}$ L. Chitu, ${ }^{1}$ M. Jergel,${ }^{1}$ S. Luby, ${ }^{1}$ A. Satka, ${ }^{2}$ and S. V. Roth ${ }^{3}$ \\ ${ }^{1}$ Institute of Physics, Slovak Academy of Sciences, Dubravska cesta 9, 84511 Bratislava, Slovakia \\ ${ }^{2}$ International Laser Center, Ilkovicova 3, 81219 Bratislava, Slovakia \\ ${ }^{3}$ HASYLAB/DESY, Notkestrasse 86, 22603 Hamburg, Germany
}

(Received 26 March 2007; published 20 November 2007)

\begin{abstract}
Evaporation of colloidal nanoparticle solutions is known to produce ordered monolayers of nanoparticles, self-assembled arrays of magnetic nanoparticles being of special importance for applications. The in situ time-resolved grazing-incidence small-angle x-ray scattering with the temporal resolution down to $100 \mathrm{~ms}$ was employed to study the self-assembling process of iron oxide nanoparticles after a colloidal drop was applied on a silicon substrate. The x-ray scattering contributions from the evaporating drop volume, drop surface, and substrate surface were monitored and separated. The x-ray scattering from the drop for the distances from the substrate surface larger than $\approx 80 \mu \mathrm{m}$ shows the absence of self-assembled clusters in the drop volume or self-assembled domains on the drop surface. These results indicate that the nanoparticle self-assembling occurs in the vicinity of the three-phase drop contact line. The ordered nanoparticle monolayer exhibits hexagonal close-packed arrangement.
\end{abstract}

DOI: 10.1103/PhysRevB.76.195432

PACS number(s): 61.10.Eq, 61.46.Df

\section{INTRODUCTION}

Nanometer-scale colloidal particles of semiconductors and noble and transition metals have attracted much attention over the two last decades due to their new electronic, magnetic, optical, and structural properties. Moreover, colloidal nanoparticles can serve as building blocks for complex thin film structures. In particular, they self-assemble into ordered two-dimensional and three-dimensional arrays (monolayers and artificial crystals, respectively) under specific conditions. Such structures shift nowadays the frontiers in advanced materials and device developments.

Ordered arrays of nanoparticles show unique behavior which is different from that of the bulk and isolated particles (see Refs. 1-4 and references therein). Various techniques making use of spontaneous self-assembling have been reported. In the simplest case, a drop of a colloidal nanoparticle solution is applied on a substrate. Subsequently, the process of solvent evaporation induces formation of single or multiple layers, resulting in an ordered nanoparticle array. Hexagonal or cubic arrangements of nanoparticles are usually observed. .,6 $^{5}$

The self-assembling is a complex process in which various interactions between nanoparticles, substrate, and solvent are involved. At the microscopic level, an interplay between localized interactions such as van der Waals attraction and hard-core (steric) repulsion (combined with long-range magnetic dipolar interaction if the particles are magnetic) determines the assembling process. However, type and thickness of the surfactant layer, interaction between the forming nanoparticle and substrate, drying kinetics, and solution/ substrate interfacial energy affect dramatically the final array formation.

For a drop of a colloidal solution deposited on a substrate, a nonzero contact angle is often observed with the contact line pinned to its initial position as the solvent evaporates. In this case, the nanoparticles move toward the contact line due to a higher evaporation rate at the drop edge. ${ }^{7}$ Also in absence of the pinning line, the convective particle flux toward substrate caused by the solvent evaporation may act in favor of the formation of ordered arrays. ${ }^{8}$ A so-called stick-slip motion of the drop contact line accompanies the formation of arrays on the substrate with more pinning centers. ${ }^{9,10}$

Model calculations of the self-assembling process based on the dynamics of the evaporating solvent indicate crucial effect of the solvent, nanoparticle size, and thermodynamic state on the morphology of the final structure ${ }^{11}$ as it was observed also experimentally. For example, formation of well ordered self-assembled arrays of dodecanthiolpassivated Au nanoparticles was achieved when the evaporation dynamics was modified by the surfactant molecules added to the colloidal solution. ${ }^{12-14}$ On the other hand, a large variety of possible resulting patterns of the selfassembled nanoparticles is a serious limitation for targeted technological applications of self-assembling. A deeper understanding of the self-assembling process, especially its initial stages, is essential for a better control of the nanoparticle ordering. This issue is closely related to answering the question where the ordering takes place. In contradiction with a common concept that the self-assembling process occurs at the substrate/colloid interface, formation of monolayer islands of dodecanthiol-passivated Au nanoparticles on the top surface of the drop (liquid/air interface) was confirmed by in situ $\mathrm{x}$-ray scattering experiments. ${ }^{13}$

In this work, we study the self-assembling process of iron oxide colloidal nanoparticles applied on silicon substrate. Magnetic iron oxide nanoparticles have great potential for applications in electronic devices and biomedicine. In situ time-resolved grazing-incidence small-angle $\mathrm{x}$-ray scattering (GISAXS) technique was employed. ${ }^{15}$ The paper is organized as follows: Sample preparation, experimental details, and theoretical background of the GISAXS calculations are described briefly in Sec. II. The results are presented, evaluated, and discussed in detail in Sec. III. Special attention is 
devoted to initial stages of the self-assembling in order to determine the region decisive for the nanoparticle ordering. Main conclusions are summarized in Sec. IV.

\section{GRAZING-INCIDENCE SMALL-ANGLE X-RAY SCATTERING EXPERIMENTS AND THEORETICAL BACKGROUND}

The iron oxide nanoparticles were synthesized by a hightemperature solution phase reaction of metal acetylacetonates $\left(\mathrm{Fe}(\mathrm{acac})_{3}\right)$ with 1,2-hexadecanediol, oleic acid, and oleylamine in phenyl ether. ${ }^{16}$ Toluene was used as a solvent. Details of the preparation procedure are described elsewhere. ${ }^{17}$ The nanoparticles are single domain due to their small size and behave as single dipoles because the dipoledipole interaction of magnetic iron oxide nanoparticles is weak. ${ }^{18}$ The nanoparticles show a well-developed crystalline structure and are superparamagnetic at room temperature, the blocking temperature being $T_{B}=22 \mathrm{~K}$. The $5 \mu \mathrm{l}$ drops of the colloid solution were deposited onto silicon substrates of $\approx 1 \mathrm{~cm}^{2}$ area covered with a native $\mathrm{SiO}_{2}$ layer. The drops were dried in air at room temperature.

The first part of our experiments was performed on the GISAXS beamline BW4 at the Hamburger Synchrotronstrahlungslabor. ${ }^{19}$ The size of the focused beam at the substrate position, as determined from $1 / e$ of the maximum intensity, was $65 \times 35 \mu \mathrm{m}^{2}$ size (horizontal $\times$ vertical). The $\mathrm{x}$-ray wavelength was set to $0.138 \mathrm{~nm}$. We employed two measurement modes. In a substrate mode, the substrate was aligned in order to halve the primary beam intensity and subsequently tilted by $0.18^{\circ}$ (standard GISAXS alignment with the angle of incidence of $0.18^{\circ}$ ). In a drop mode, the substrate was vertically translated downward by $100 \mu \mathrm{m}$ out of the primary beam after having been aligned and subsequently tilted by $0.1^{\circ}$ in order to eliminate the x-ray scattering from the substrate. In this mode, solely the x-ray scattering from the drop (including its surface as it was crossing the primary beam during evaporation) was measured. The scattered x-ray radiation was detected by a two-dimensional x-ray charge coupled device (CCD) camera located at the distance of $1971 \mathrm{~mm}$ from the substrate. Each CCD pattern was acquired for $2.6 \mathrm{~s}$ if not stated otherwise.

The second part of our measurements was performed on a horizontal x-ray diffractometer (Bruker D8 Discover Super Speed Solution) equipped with an $18 \mathrm{~kW} \mathrm{Cu}$ rotating anode TXS generator (wavelength of $0.154 \mathrm{~nm}$ ). A parabolic Goebel mirror provided the primary beam of $0.03^{\circ}$ full width at half maximum horizontal divergence and $10^{9}$ photons/s intensity. The horizontal and vertical beam sizes were restricted to $1 \mathrm{~mm}$ with a pinhole collimator. A knife-edge collimator was translated downward to the substrate surface in order to suppress the superfluous primary beam but not to influence the intensity of the useful GISAXS signal. The incident angle of $0.2^{\circ}$ was adjusted by tilting the sample stage. A beamstop beyond the sample stage absorbed the passing primary beam. A Soller slit of $0.35^{\circ}$ horizontal angular acceptance and a point scintillation detector set at a fixed angle were used to collect the GISAXS signal with a $100 \mathrm{~ms}$ temporal resolution. Using the laboratory source, we

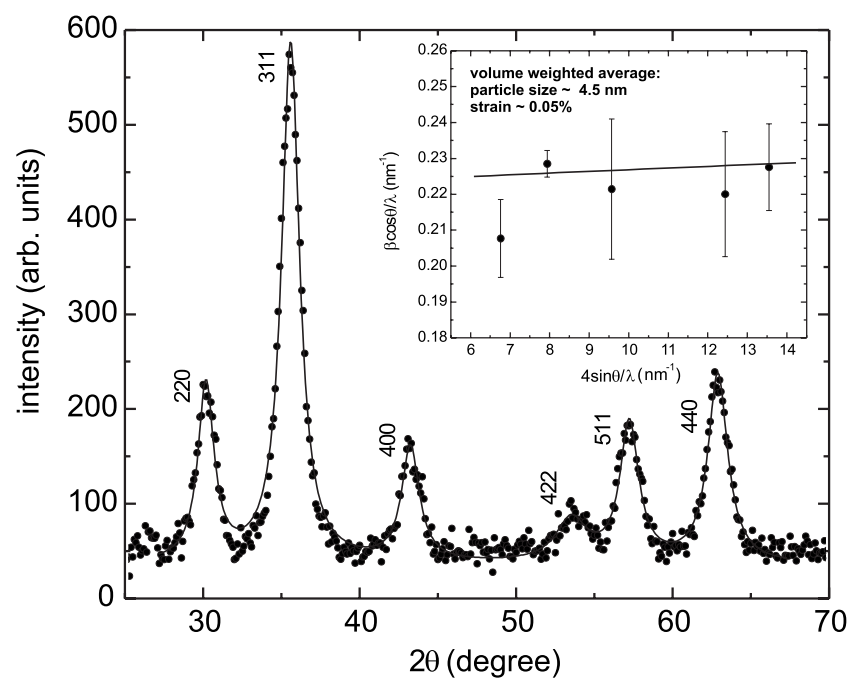

FIG. 1. Grazing-incidence x-ray diffraction pattern of a multilayer stack of iron oxide nanoparticles on silicon substrate measured at $1.5^{\circ}$ angle of incidence. The inset shows a WilliamsonHall plot based on positions and widths of the measured diffraction peaks.

were able to measure the GISAXS signal few seconds after the deposition of the drop and to follow the early stages of the assembling process.

Distorted-wave Born approximation (DWBA) proved to be a convenient approach to calculate the GISAXS from nanoparticles deposited onto a planar surface..$^{20-22}$ Briefly, a semi-infinite substrate represents an undisturbed system and freestanding particles on it introduce a disturbance. The observed GISAXS intensity corresponds to the diffuse part of the total differential scattering cross section calculated for the disturbed system, which results in a product of a single particle form factor related to the nanoparticle shape and an interference function related to the nanoparticle arrangement. For our GISAXS simulation, a bidimensional paracrystal model $^{23}$ was applied to describe an array of spherical nanoparticles. In this model, the long-range order is destroyed gradually in a probabilistic way. Further details of the GISAXS calculations as performed in this work may be found in Refs. 24 and 25.

\section{RESULTS: EVALUATION AND DISCUSSION}

\section{A. Structural properties and arrangement of iron oxide nanoparticles}

Structure of the synthesized iron oxide nanoparticles was inspected by grazing-incidence $x$-ray diffraction at $1.5^{\circ}$ angle of incidence. Multiple layers of iron oxide nanoparticles were obtained by the evaporation of a concentrated colloidal solution at room temperature. The diffraction pattern is shown in Fig. 1. Positions and relative intensities of the diffraction peaks match those tabulated for powder diffraction pattern of magnetite $\left(\mathrm{Fe}_{3} \mathrm{O}_{4}\right) .{ }^{26}$ The diffraction peaks were fitted by the Lorentzian profile in order to estimate the particle size and microstrain from a Williamson-Hall plot $^{27}$ 


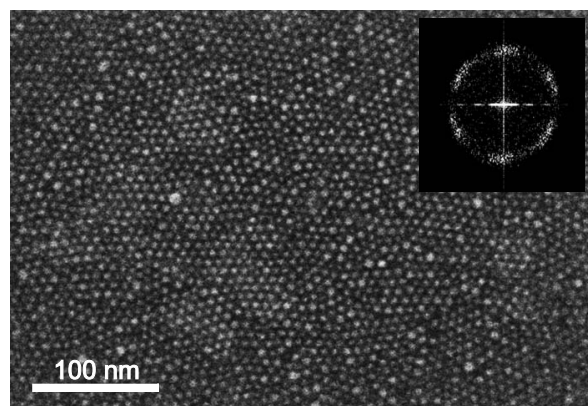

FIG. 2. Scanning electron microscope image of an iron oxide nanoparticle monolayer on silicon substrate. Fourier transform of the image shown in the inset reveals hexagonal symmetry of the self-assembling.

(inset in Fig. 1). The volume-weighted average particle diameter is $D_{V}=4.5 \pm 0.3 \mathrm{~nm}$ and the residual microstrain is $\varepsilon$ $=0.05 \%$. For monodisperse spherical particles, $D_{V}=(3 / 4) D$, where $D$ is the particle diameter. ${ }^{28}$ Using this relation, the average particle diameter estimated by the $\mathrm{x}$-ray diffraction is $6.0 \pm 0.4 \mathrm{~nm}$. The average particle diameter calculated from the transmission electron microscopy (JEM 100C) is $5.5 \pm 0.9 \mathrm{~nm}$, which is in good agreement with the diffraction result.

Monolayers of the iron oxide nanoparticles were prepared by the solvent evaporation from the drop of the colloidal solution applied on the substrate at room temperature. According to Ref. 29, three distinct stages of drying the drop are as follows: After the application on the surface, the radius of the drop increases and the drop wets the substrate. After reaching the maximum diameter, the contact line stays pinned for a certain time - the solvent evaporation and the wetting of substrate are in balance. In the second stage, the evaporation moves the contact line in the stick-slip manner. The first 1-2 min can be characterized by a linear mass decrease of $\approx 45 \mu \mathrm{g} / \mathrm{s}$. In the third stage, the evaporationdriven surface tension instability ${ }^{30}$ results in a fast drop migration over the substrate. The drop is completely dried within $\approx 5 \mathrm{~min}$.

A representative image of the self-assembled monolayer acquired by scanning electron microscope (FEG Leo 1550) is shown in Fig. 2. The inset shows the Fourier transform of the entire image. It can be seen that the nanoparticles are ordered in a perfect hexagonal close-packed array within the domains of an apparent size less than $100 \mathrm{~nm}$. A partial smearing out of the reciprocal lattice points is a consequence of mutually misaligned domains. The average particle size obtained from Fig. 2 is $6.4 \pm 0.6 \mathrm{~nm}$, which is slightly larger than that determined by the transmission electron microscopy. This is mainly due to the fact the scanning electron microscope is more sensitive to the organic surfactant shell surrounding the iron oxide particle core. The average interparticle distance (center-to-center) was found to be $7.7 \pm 0.6 \mathrm{~nm}$.

\section{B. Time-resolved grazing-incidence small-angle $x$-ray scattering studies with synchrotron radiation}

A typical GISAXS pattern of the nanoparticle array on silicon substrate taken in the substrate mode after the solvent
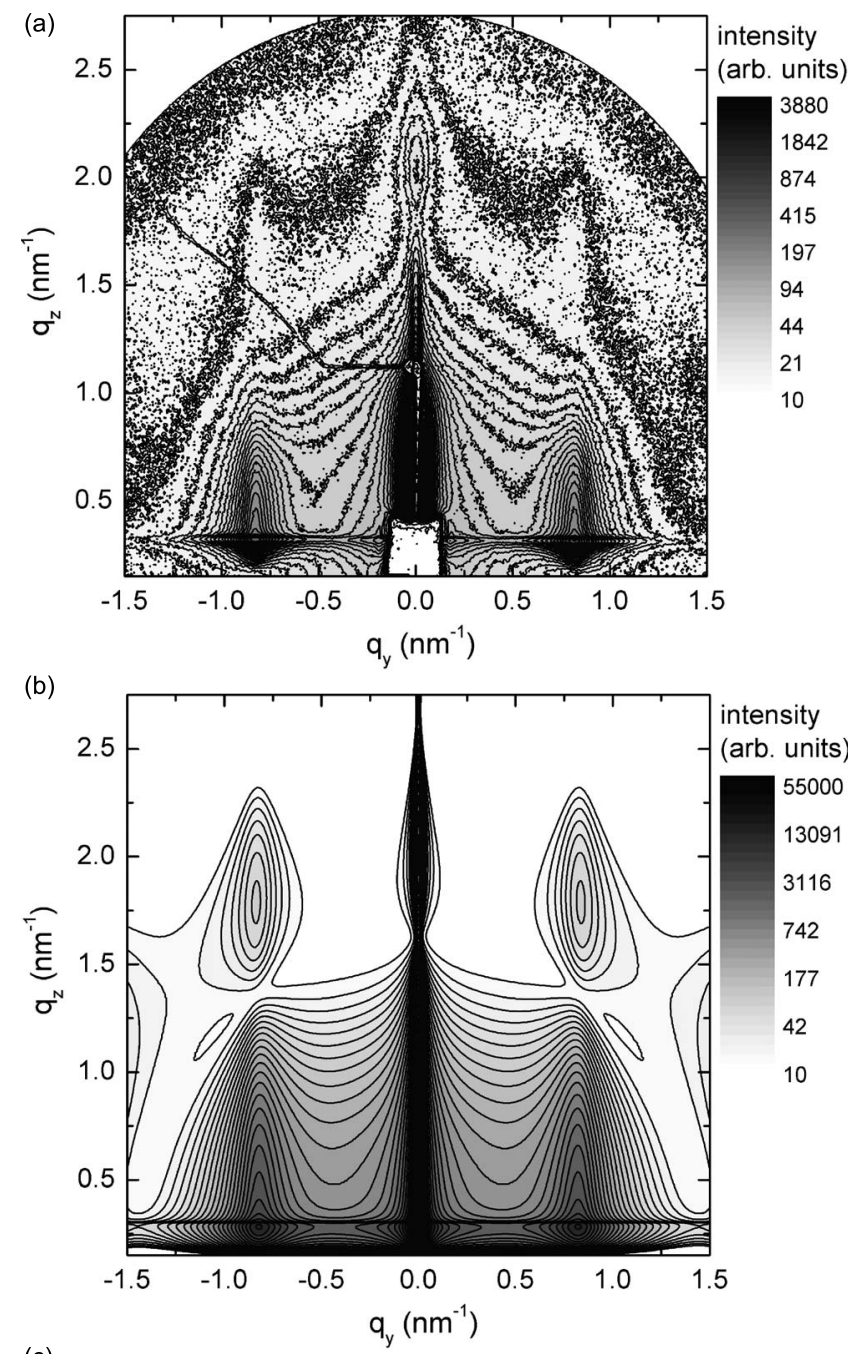

(c)

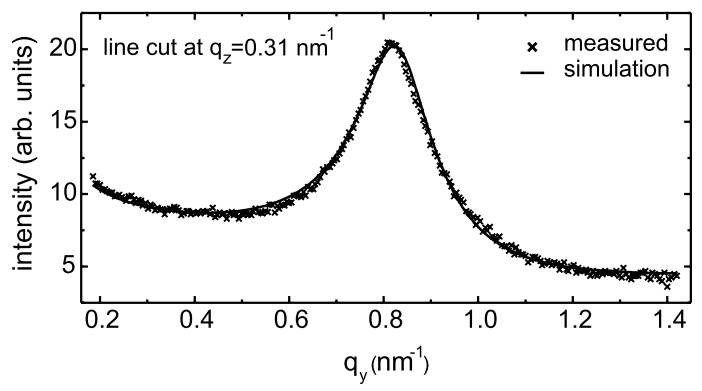

FIG. 3. (a) Measured and (b) simulated GISAXS patterns of the self-assembled nanoparticle array and (c) a fit of a line cut of the measured GISAXS pattern along $q_{y}$ at $q_{z}=0.31 \mathrm{~nm}^{-1}$ (dots, measured points; line, fit). The central part along $q_{z}$ is shadowed by a beamstop.

evaporation is shown in Fig. 3(a). The $q_{y}$ and $q_{z}$ components of the scattering vector are parallel and perpendicular to the substrate surface, respectively. The specularly reflected beam and diffuse scattering along $q_{z}$ for $q_{y}=0$ were blocked by a beamstop to avoid the CCD camera saturation. The empty areas in the left and right upper corners are due to the detection absence. The observed GISAXS pattern is given by an interference of the diffusely scattered $x$ rays from each irra- 

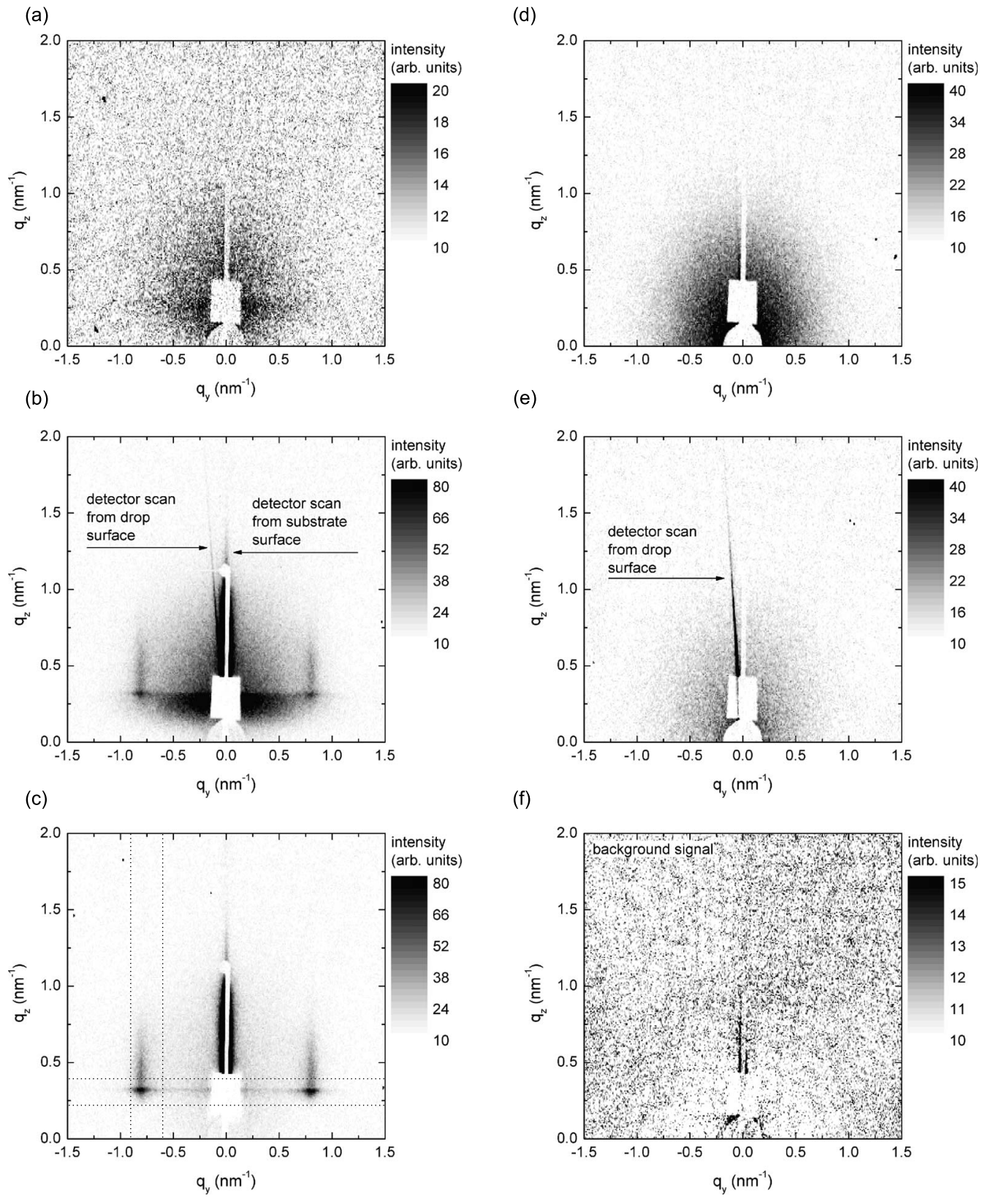

FIG. 4. GISAXS patterns taken in the $[(\mathrm{a})-(\mathrm{c})]$ substrate and $[(\mathrm{d})-(\mathrm{f})]$ drop modes measured at different stages of the drop evaporation.

diated nanoparticle in the ordered array. The side maxima at $q_{y} \approx \pm 0.82 \mathrm{~nm}^{-1}$ indicate the interparticle spacing of $2 \pi / q_{y}$ $\approx 7.7 \mathrm{~nm}$, in agreement with the scanning electron microscopy.

The simulation of the measured GISAXS pattern was performed using the DWBA and bidimensional paracrystal model as mentioned above. The simulation and a selected line cut at the critical exit angle $\left(q_{z}=0.31 \mathrm{~nm}^{-1}\right)$ are shown in Figs. 3(b) and 3(c), respectively. It can be seen that all measured features are well reproduced. From fitting the line cut, the following parameters were obtained: the average particle diameter of $6.1 \pm 0.6 \mathrm{~nm}$, the average interparticle distance of $7.5 \pm 1 \mathrm{~nm}$, and the lateral correlation length of the particle distribution of $87 \mathrm{~nm}$. All these values are within the confidence intervals of the corresponding values determined by the x-ray diffraction and transmission and scanning electron microscopies.

In situ temporal evolution of the GISAXS pattern was studied both in the substrate and drop modes. Three typical stages of the temporal evolution in the substrate mode are shown in Figs. 4(a)-4(c). The first stage is characterized by the x-ray scattering from the volume of the colloidal drop, the drop being larger than the beam size [Fig. 4(a)]. Due to a high x-ray absorption coefficient of the colloidal solution, only a small part of the incoming radiation penetrates the drop and reaches the detector. The signal detected is featureless and the intensity decreases monotonously along $q_{y}$ and $q_{z}$ axes. No ordering of the nanoparticles in the solution is 
observed. In an intermediate stage of the solvent evaporation, the drop surface is crossing the incident $\mathrm{x}$-ray beam and the surface scattering from the already dried nanoparticle array on the substrate as well as the volume and surface scattering from the still evaporating drop are observed. Maxima at $q_{y} \approx \pm 0.8 \mathrm{~nm}^{-1}$ appearing in this stage indicate a nanoparticle ordering on the substrate and/or the drop surface [Fig. 4(b)]. The GISAXS maxima are well resolved in Fig. 4(c), which corresponds to an ordered array of nanoparticles after drying the drop. Moreover, we can see two stripes in the GISAXS pattern [marked with arrows in Fig. 4(b)], which can be understood as projections of the detector scans (in the language of the coplanar measurements with a point detector) in the $q_{x}-q_{z}$ plane onto the $q_{y}-q_{z}$ plane of the CCD camera $\left(q_{x}\right.$ is more than 1 order of magnitude smaller than $q_{z}$ ). The stripe parallel to $q_{z}$ comes from the scattering from the substrate surface and can be observed also in Fig. 4(c) for the dried array of nanoparticles. The other stripe comes from the scattering from the drop surface. Though this surface is curved, the stripe is not fanned out because of the local surface sampling by the microfocus beam. The nonzero angle between the two stripes depends on mutual orientation of the respective surface normals. The shape of the drop surface is a complex function of time and may vary from drop to drop as a result of the statistical nature of the evaporation dynamics.

The GISAXS results obtained in the drop mode are shown in Figs. 4(d)-4(f). The first stage is dominated by the volume scattering from the colloidal drop [Fig. 4(d)]. The signal decreases monotonously with increasing scattering vector in the reciprocal space. Similar as in Fig. 4(b), an abrupt change of the GISAXS pattern is observed when the drop surface is crossing the incident X-ray beam [Fig. 4(e)]. Simultaneously, one sharp stripe (indicated by an arrow), which corresponds to the scattering from the drop surface as explained above, appears. The GISAXS signal comes from the drop volume and drop surface but no specific features indicating a nanoparticle ordering are observed. After the drop surface passed the incident beam, only a background signal is detected [Fig. 4(f)].

In order to visualize the temporal evolution of the GISAXS, we used $t-q_{y}$ intensity maps. The intensity corresponding to a particular $\left(t, q_{y}\right)$ point is obtained by an integration of the measured intensity over the $q_{z}$ interval of $\left\langle 0.22 \mathrm{~nm}^{-1}, 0.39 \mathrm{~nm}^{-1}\right\rangle$ [marked by the horizontal dotted lines in Fig. 4(c)] at a constant $q_{y}$ in the GISAXS pattern taken at the time instant $t$ after starting the detection at $t=0$. This zero time is shifted by $\approx 30 \mathrm{~s}$ with respect to the first contact of the drop with the substrate surface so that the temporal scale used is relative. Being not interested in the duration of the evaporation process itself, the absolute time is of little importance for us. In order to quantify the temporal evolution, we integrated further the scattered intensity also over the $q_{y}$ interval of $\left\langle 0.6 \mathrm{~nm}^{-1}, 0.9 \mathrm{~nm}^{-1}\right\rangle$ to obtain a partial integrated scattering (PIS) as a function of time. The PIS plot shows the temporal evolution of the GISAXS intensity integrated over the area marked by the vertical and horizontal dotted lines in Fig. 4(c) where the side maximum appears.

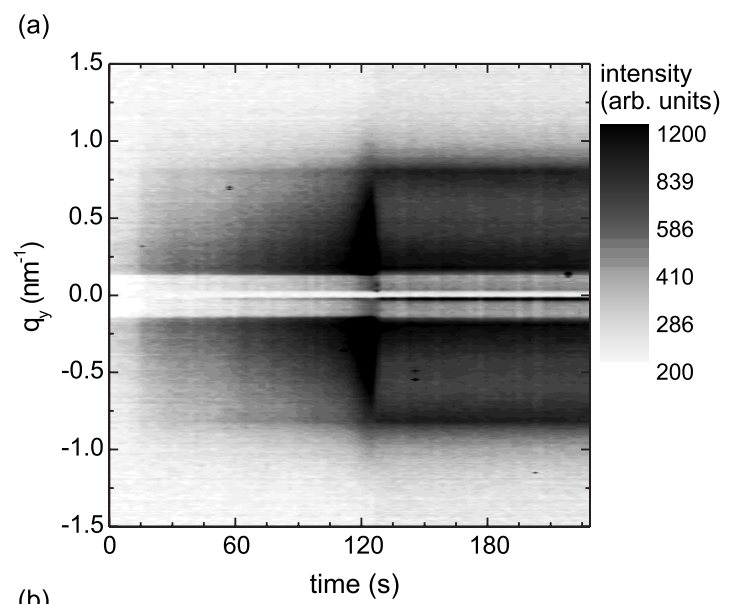

(b)

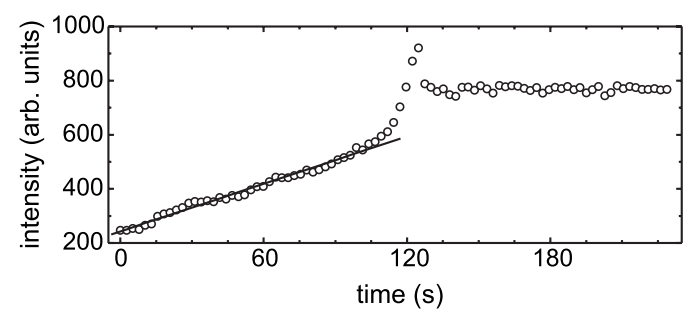

FIG. 5. (a) A $t$ - $q_{y}$ map of a drying colloidal drop forming a monolayer of ordered nanoparticles measured in the substrate mode and (b) the corresponding PIS plot.

Figure 5(a) shows the $t-q_{y}$ map measured in the substrate mode. The GISAXS signal comes from the already dried nanoparticles on the substrate, the drop volume, and drop surface. Distinct lines at $q_{y} \approx \pm 0.8 \mathrm{~nm}^{-1}$ are observable from the early stages, indicating the formation of an ordered nanoparticle array from the very beginning of the detection. The GISAXS signal along the $q_{y}$ direction increases gradually with time and culminates at $t \approx 120 \mathrm{~s}$ when the drop surface crosses and scatters strongly the x-ray beam in the form of a detector scan as explained above [compare with Fig. 4(b)]. The corresponding PIS plot is shown in Fig. 5(b). At the beginning, the scattered signal grows linearly, while an exponential-like increase is observed when the drop surface crosses the primary $\mathrm{x}$-ray beam before escaping from it completely. After this transient, the scattered intensity decreases to a stationary value produced solely by the dried selfassembled nanoparticle array on the substrate.

The overall initial increase of the GISAXS signal in the $t-q_{y}$ map is a result of the steadily increasing nanoparticle concentration in the drop volume as the solvent evaporates. For the PIS plot related to the region of the side maximum in the reciprocal space, the growing area of ordered nanoparticles on the substrate surface may play a role as well. In order to estimate the contribution of the scattering from the drop volume to the total scattered intensity during the drying process, we repeated the temporal analysis with the colloidal solution diluted by a factor of 10 . The $t-q_{y}$ map and PIS plot are shown in Figs. 6(a) and 6(b), respectively. The GISAXS signal in the $t-q_{y}$ map increases with time up to a maximum at $t \approx 120 \mathrm{~s}$ before the drop escapes from the $\mathrm{x}$-ray beam and a steady-state regime is established. Ordering lines at $q_{y}$ 
(a)

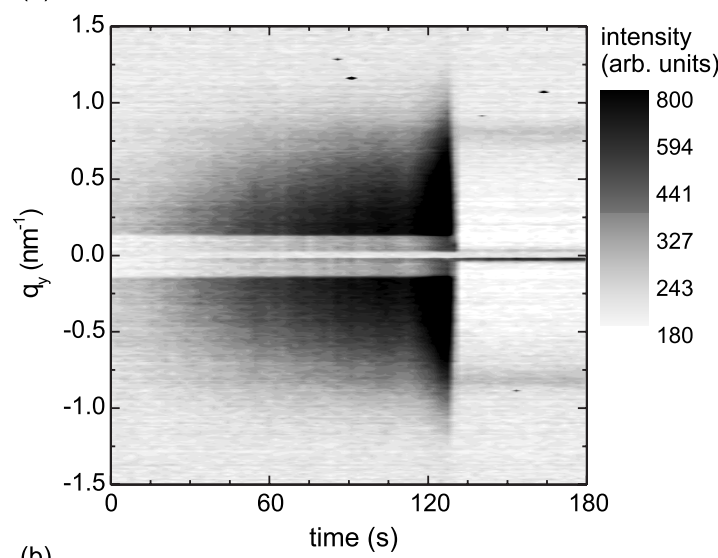

(b)

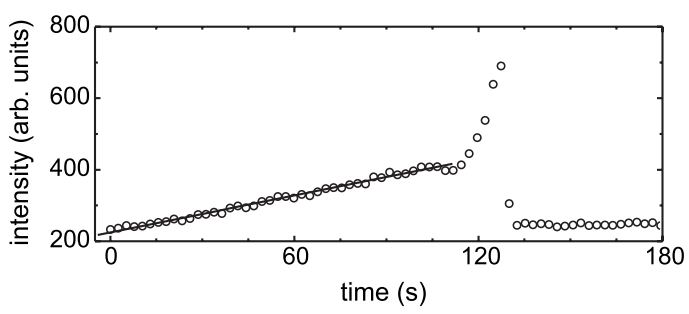

FIG. 6. (a) A $t$ - $q_{y}$ map of a drying diluted colloidal drop forming ordered submonolayer arrays of nanoparticles measured in the substrate mode and (b) the corresponding PIS plot.

$\approx \pm 0.8 \mathrm{~nm}^{-1}$ can be resolved in the steady-state regime but their traces are visible already at earlier stages. A submonolayer coverage of the substrate consisting of isolated islands of the ordered nanoparticle arrays was observed by the scanning electron microscopy after drying the drop. The islands produce a smaller PIS signal than the monolayer. The PIS temporal plot exhibits again a linear increase up to $t$ $\approx 100 \mathrm{~s}$ followed by a transient and steady-state regions, the linear signal being higher than the steady-state one coming from the ordered nanoparticle array on the substrate. This fact indicates clearly that also in the PIS plot, the main contribution to the scattered intensity before the drop escapes from the x-ray beam comes from the nanoparticles inside the drop.

In view of these findings, the initial linear increase of the GISAXS signal can be attributed to the linear increase of the nanoparticle concentration as the drop is losing its mass during the solvent evaporation. The amount of the deposited nanoparticles is still negligible when compared to the number of the nanoparticles inside the drop. The growing nanoparticle concentration leads to the observed linear growth of the volume and surface scattering from the drop. Later, the evaporating drop surface crosses the x-ray beam and the drop position becomes unstable. Finally, the drop moves completely out of the x-ray beam and an abrupt intensity decrease resulting in a stationary time-independent GISAXS signal is observed in the PIS plot. This decrease is not connected with a loss of the nanoparticle order but with the absence of the drop scattering.

It still remains to determine the region of the nanoparticle self-assembling. Measurements in the substrate mode cannot (a)

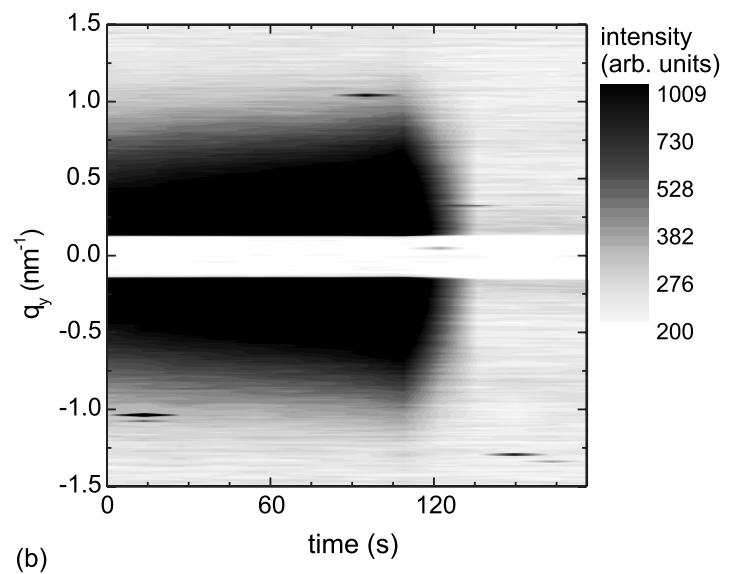

(b)

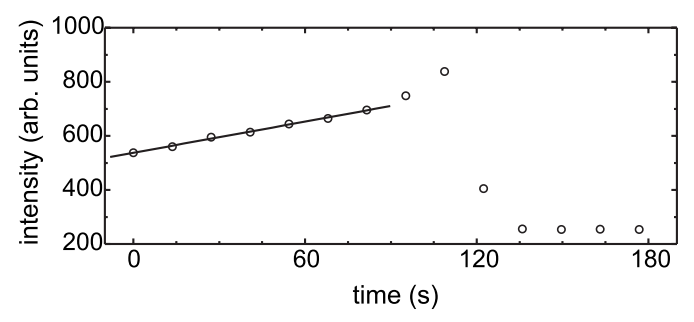

FIG. 7. (a) $t-q_{y}$ map of a drying colloidal drop measured in the drop mode and (b) the corresponding PIS plot.

distinguish between the signal scattered from the ordered nanoparticle assembly on the substrate surface and on the drop surface. Therefore, we utilized the drop mode which probes the drop exclusively. In order to minimize the impact of the readout time on possible short transients, we enhanced the integration time of a single GISAXS pattern from 2.6 to $13.6 \mathrm{~s}$. The measured $t-q_{y}$ map and PIS plot are shown in Figs. 7(a) and 7(b), respectively. Different from the substrate mode, the $t-q_{y}$ map shows no ordering lines and the GISAXS signal along $q_{y}$ decays monotonously with time up to $t \approx 120 \mathrm{~s}$. The linear increase of the PIS plot is dominated by the volume scattering of the linearly densifying drop. The exponential-like final increase may be attributed to a combined effect of the volume and surface scattering from the colloidal drop [compare with Fig. 4(e)]. Finally, the drop escapes from the x-ray beam and the measured scattering signal goes to a background value. No evidence of the existence of ordered nanoparticle arrays in the drop volume or on the drop surface within the employed statistics has an important implication. According to our calculations, $\approx 63 \%$ of the incident $\mathrm{x}$-ray intensity probed the drop volume located between 82 and $118 \mu \mathrm{m}$ above the substrate surface. Therefore, the self-assembly process takes place no more than $\approx 80 \mu \mathrm{m}$ above the substrate surface in the vicinity of the drop contact line. The contact line moves steadily in the stick-slip manner and the nanoparticles, which are ordering in its neighborhood, adhere to the substrate surface as the solvent evaporates.

\section{Time-resolved grazing-incidence small-angle $x$-ray scattering studies with $x$-ray rotating anode source}

A poor temporal resolution poses a risk of data undersampling when monitoring fast dynamical processes. In order to 


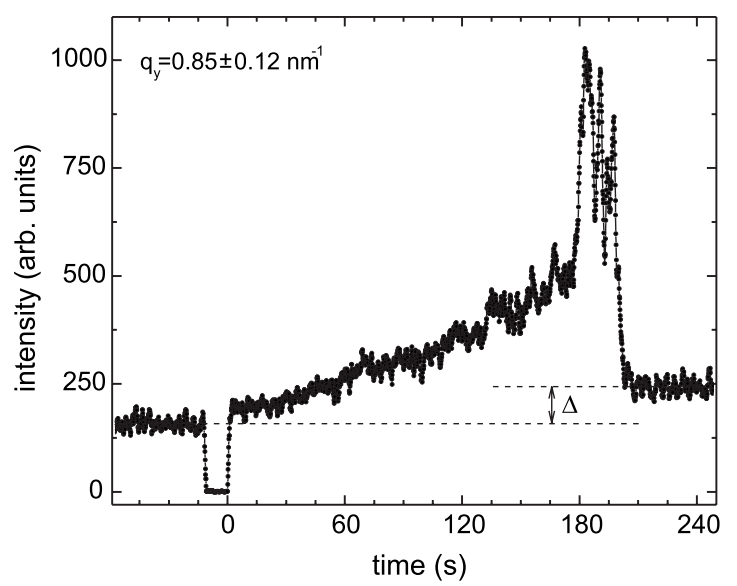

FIG. 8. PIS plot of a drying colloidal drop in the substrate mode measured with $\mathrm{x}$-ray rotating anode source.

avoid deceptive results, we measured the GISAXS at a fixed scattering angle using a rotating anode $\mathrm{x}$-ray source. The GISAXS signal was sampled at $10 \mathrm{~Hz}$, i.e., 26 times faster than it was possible with the x-ray CCD camera. In this way, we were able to study the early stages of the self-assembling process. A horizontal Soller slit was positioned parallel to the substrate normal so that the measured GISAXS intensity was integrated along the $q_{z}$ direction and convoluted with the slit acceptance along the $q_{y}$ direction. A PIS plot integrated over the $q_{y}$ interval of $\left\langle 0.73 \mathrm{~nm}^{-1}, 0.97 \mathrm{~nm}^{-1}\right\rangle$ is shown in Fig. 8 . The zero time $t=0$ refers to the start of the detection after the drop was applied on the substrate. For negative times, before the drop was applied, we observe diffuse scattering from the clean substrate. The application of the drop is displayed by a missing part of the signal. The drop evaporation is accompanied by a linear increase of the scattered intensity up to $t$ $\approx 120 \mathrm{~s}$, as observed also by the time-resolved synchrotron measurements. This evaporation phase is dominated by an increasing volume scattering from the densifying colloidal drop, a small contribution of the already dried ordered nanoparticle array on the substrate being also possible. After this linear increase, several fast fluctuating transients are observed. These transients are a consequence of the evaporation-driven surface tension instability and vary from drop to drop. The drying drop with a fast modifying surface moves into and out of the x-ray beam and the contributions of the volume and surface scattering from the drop produce "revivals" of the GISAXS signal accordingly. These transients were undersampled in the CCD synchrotron measurements, resulting in the smooth exponential-like increase of the PIS plot. The difference between the initial and final PIS levels (denoted by the symbol $\Delta$ in Fig. 8) shows a contribution of the dried self-assembled nanoparticles to the GISAXS signal.

\section{CONCLUSIONS}

The self-assembling of colloidal nanoparticles was studied by the time-resolved GISAXS measurements using both synchrotron radiation and a conventional $x$-ray rotating anode source. The contribution of the volume and surface x-ray scattering from the drop to the total GISAXS signal during the self-assembling was separated in the synchrotron measurements. The focused $\mathrm{x}$-ray beam provided the temporal evolution of the volume and surface scattering from the drop, being not disturbed by the substrate scattering and giving thus an insight into possible nanoparticle self-assemblies forming on the drop surface or inside the drop. The colloidal nanoparticle solution used shows the absence of selfassembled clusters in the drop volume or self-assembled domains on the drop surface for the distances from the substrate surface larger than $\approx 80 \mu \mathrm{m}$. Relying on these results, we assume that the self-assembling takes place in the vicinity of the three-phase (liquid-solid-air) drop contact line as the solvent evaporates (i.e., not inside or on the drop). The dried nanoparticle monolayer exhibits hexagonal close-packed arrangement. Similar measurements with a much better temporal resolution enabling us to monitor also the early stages of the self-assembling process were performed with the X-ray rotating anode. Fast transients of the GISAXS signal in the final evaporation stage indicate a highly nonlinear behavior of the volume and surface $\mathrm{x}$-ray scattering as a result of the evaporation-driven surface tension instability of the drying drop.

\section{ACKNOWLEDGMENTS}

This work was supported by Scientific Grant Agency VEGA Bratislava, Grant No. 2/6030/26, by Centre of Excellence SAS Physics of Information Contract No. I/2/2007, and by Slovak Research and Development Agency Grants Nos. APVV-LPP-0080-06 and APVV-0173-06.
${ }^{1}$ C. P. Collier, T. Vossmeyer, and J. R. Heath, Annu. Rev. Phys. Chem. 49, 371 (1998).

${ }^{2}$ C. B. Murray, C. R. Kagan, and M. G. Bawendi, Annu. Rev. Mater. Sci. 30, 545 (2000).

${ }^{3}$ E. V. Shevchenko, D. V. Talapin, H. Schnablegger, A. Kornowski, O. Festin, P. Svedlinh, M. Haase, and H. Weller, J. Am. Chem. Soc. 125, 9090 (2003).

${ }^{4}$ C. Petit, A. Taleb, and M. P. Pileni, Adv. Mater. (Weinheim, Ger.) 10, 259 (1998).
${ }^{5}$ M. D. Bentzon and A. Tholen, Ultramicroscopy 38, 105 (1990).

${ }^{6}$ C. B. Murray, C. R. Kagan, and M. G. Bawendi, Science 270, 1335 (1995).

${ }^{7}$ R. D. Deegan, O. Bakajin, T. F. Dupont, G. Huber, S. R. Nagel, and T. A. Witten, Nature (London) 389, 827 (1997); Phys. Rev. E 62, 756 (2000).

${ }^{8}$ N. D. Denkov, O. D. Velev, P. A. Kralchevsky, I. B. Ivanov, H. Yoshimura, and K. Nagayama, Nature (London) 361, 26 (1993).

${ }^{9}$ E. Adachi, A. S. Dimitriov, and K. Nagayama, Langmuir 11, 
1057 (1995).

${ }^{10}$ L. Shmuylovich, A. Q. Shen, and H. A. Stone, Langmuir 18, 3441 (2002).

${ }^{11}$ E. Rabani, D. R. Reichman, Ph. L. Geissler, and L. E. Brus, Nature (London) 426, 271 (2003).

${ }^{12}$ X. M. Lin, H. M. Jaeger, C. M. Sorensen, and K. J. Klabunde, J. Phys. Chem. B 105, 3353 (2001).

${ }^{13}$ S. Narayanan, J. Wang, and X. M. Lin, Phys. Rev. Lett. 93, 135503 (2004).

${ }^{14}$ T. P. Bigioni, X. M. Lin, T. T. Nguyen, E. I. Corwin, T. A. Witten, and H. M. Jaeger, Nat. Mater. 5, 265 (2006).

${ }^{15}$ G. Renaud, R. Lazzari, C. Revenant, A. Barbier, M. Noblet, O. Ulrich, F. Leroy, J. Jupille, Y. Borensztein, C. R. Henry, J. P. Deville, F. Scheurer, J. Mane-Mane, and O. Fruchart, Science 300, 1416 (2003).

${ }^{16}$ Sh. Sun, H. Zeng, D. B. Robinson, S. Raoux, Ph. M. Rice, Sh. X. Wang, and G. Li, J. Am. Chem. Soc. 126, 273 (2004).

${ }^{17}$ L. Chitu, M. Jergel, E. Majkova, S. Luby, I. Capek, A. Satka, J. Ivan, J. Kovac, and M. Timko, Mater. Sci. Eng., C 27, 1415 (2007).

${ }^{18}$ Y. Lalatone, J. Richardi, and M. P. Pileni, Nat. Mater. 3, 121 (2003).

${ }^{19}$ S. V. Roth, R. Döhrmann, M. Dommach, M. Kuhlmann, I.
Kröger, R. Gehrke, H. Walter, C. Schroer, B. Lengeler, and P. Müller-Buschbaum, Rev. Sci. Instrum. 77, 085106 (2006).

${ }^{20}$ M. Rauscher, T. Salditt, and H. Spohn, Phys. Rev. B 52, 16855 (1995).

${ }^{21}$ M. Rauscher, R. Paniago, H. Metzger, Z. Kovats, J. Domke, J. Peisl, H. D. Pfannes, J. Schulze, and J. Eisele, J. Appl. Phys. 86, 6763 (1999).

${ }^{22}$ J. Stangl, V. Holý, T. Roch, A. Daniel, G. Bauer, J. Zhu, K. Brunner, and G. Abstreiter, Phys. Rev. B 62, 7229 (2000).

${ }^{23}$ R. Hosemann, W. Vogel, and D. Weick, Acta Crystallogr., Sect. A: Cryst. Phys., Diffr., Theor. Gen. Crystallogr. 37, 85 (1981).

${ }^{24}$ R. Lazzari, J. Appl. Crystallogr. 35, 406 (2002).

${ }^{25}$ Y. Chushkin, M. Ulmeanu, S. Luby, E. Majkova, I. Kostic, P. Klang, V. Holy, Z. Bochnicek, M. Giersig, M. Hilgendorff, and T. H. Metzger, J. Appl. Phys. 94, 7743 (2003).

${ }^{26}$ The International Centre for Diffraction Data (ICDD) Database, Data Set File No. 00-019-0629.

${ }^{27}$ G. K. Williamson and W. M. Hall, Acta Metall. 1, 22 (1954).

${ }^{28}$ N. C. Popa and D. Balzar, J. Appl. Crystallogr. 35, 338 (2002).

${ }^{29}$ C. Poulard, G. Guéna, and A. M. Cazabat, J. Phys.: Condens. Matter 17, S4213 (2005).

${ }^{30}$ S. G. Yiantsios and B. G. Higgins, Phys. Fluids 18, 082103 (2006). 